\title{
Identidad profesional: proceso de configuración en el caso de dirigentes de organizaciones no gubernamentales
}

\author{
Erico Rentería ${ }^{1}$ e Carlos Fernando Torres ${ }^{2}$ \\ Instituto de Psicología, Universidad del Valle (Cali, Colombia)
}

\begin{abstract}
Considerando nuevas formas de relacionamiento entre personas y trabajo que emergen a partir de cambios en el mundo social, político y económico propios de la llamada postmodernidad, la investigación presentada caracteriza a partir de narrativas referidas a las trayectorias personales y profesionales, la configuración de la identidad de dirigentes de Organizaciones No Gubernamentales, con el propósito de contribuir a la ampliación de los repertorios de comprensión psicosocial, que faciliten la lectura sobre realidades actuales del trabajo en el contexto colombiano, diferentes a las de organizaciones de trabajo de tipo empresarial. Se utilizaron entrevistas en profundidad, en cuatro ciudades, procesadas mediante análisis de contenido y ampliadas con la técnica de líneas narrativas, a partir del abordaje teórico-metodológico de prácticas discursivas y producción de sentidos en el cotidiano. Se detectaron cinco grandes fases en las trayectorias personales-profesionales desde la noción de posicionamiento, lo que permitió perfilar el desarrollo de la carrera a partir de identificaciones, diferenciaciones y rupturas a lo largo de los relatos de la propia vida.
\end{abstract}

Palabras-clave: Trabajo, Identidad, Trayectoria, Carrera, Posicionamientos, ONG.

Professional identity: configuration process for leaders of non-governmental organizations

Considering the new forms of relation between people and their work which emerge from social, political and economical changes resulting from the so called "post modernity", this research is characterized by narratives referring to personal life and professional career, the configuration of identity of non-governmental organization leaders, aiming to contribute to the expansion of repertoires of psychosocial comprehension that allow our reading of current realities of work in the Colombian context, which differ from work organizations of business type. Interviews made in four cities were deeply used, processed through content analysis and widened through the technique of narrative line, using a theoretical-methodological approach of narrative practices and the production of meaning in daily life. Five big stages along the personal and professional lives were detected, beginning at the positioning concept, which allowed us to outline the development of a career based on identifications, differentiation and ruptures all throughout the narrative of one's own life.

Keywords: Work, Identity, Trajectory, Career, Positioning, NGO.

\section{Introducción}

T a pertinencia actual del concepto de identidad profesional viene siendo discutida por Lacadémicos del mundo del trabajo (Bauman, 2005; Beck \& Beck-Gernsheim, 2002; Offe, 1995; Sato, 1997, 2002; Malvezzi, 2000, 2001; Sennett, 2000, 2002; Rentería, 2008, 2009; Ribeiro, 2009) proponiendo elementos que han ido esclareciendo dimensiones subjetivas en relación con los cambios sociales, políticos, económicos y culturales a los que asistimos desde la segunda mitad del siglo XX. En este sentido, se retoman los aportes de algunos sociólogos que han propuesto reflexiones acerca de las nuevas formas de identidad considerando los procesos históricos de las sociedades occidentales. Así por su parte, Bauman (2005) hace referencia a la

1 Profesor, Instituto de Psicología, Universidad del Valle, Cali, Colombia. Doctor en Psicología pela Universidad de São Paulo, Brasil. Director del Grupo de Investigación en Psicología Organizacional y del Trabajo, Instituto de Psicología, Universidad del Valle.

2 Magister en Psicología, línea de formación en psicología organizacional y del trabajo, Universidad del Valle, Cali, Colombia. Profesor ocasional en Facultad de Ciencias de la Administración, Universidad del Valle. 
pérdida progresiva de la solidez que caracterizó las instituciones tradicionales con las que se organizó la vida social, en otras palabras, dimensiones como la familia o el trabajo, tienden a dejar de ser "moldes" que proporcionaban coordenadas de identidades claras y concretas para las personas, pasando a ser en la actualidad "categorías zombis", que sobreviven como instituciones pero que ya no son tomadas estrictamente como referentes identitarios en un sentido de estabilidad. De otro lado, Beck y Beck-Gernsheim (2002) parten de debatir sobre la tendencia de las instituciones sociales hacia la desarticulación de proyectos colectivos en lo que denominan "proceso de individualización". De este modo, para ellos el sujeto posmoderno se encuentra ante la relativa posibilidad de decidir sobre su propia vida, sin tener que acudir necesariamente a aquellas colectividades representadas tradicionalmente por el Estado, la comunidad o la familia, trazando su propia trayectoria a partir de soluciones biográficas.

Los cambios que presenciamos en el mundo de los negocios, han ido impactando múltiples ámbitos en los que se observan rasgos de flexibilidad, rapidez y diversidad de formas organizativas mediadas por lo subjetivo (Rentería, 2008). En este contexto el nuevo trabajador, como sujeto histórico, responde a partir de sus propios recursos (Rentería \& Malvezzi, 2008) ante las demandas del medio y a favor de lo que considera como sus propias necesidades e intereses. Malvezzi (1999) sugiere la noción de agente económico reflexivo, como aquel profesional que tiene la crítica y la hermenéutica como sus principales herramientas, y cuya identidad ya no está dada por estructuras que lo superan para trazarle el trayecto de su vida (Schein, 1982) de trabajo, ligada a una única organización. En ese sentido estamos ante una concepción de persona que trabaja, que asume su carácter dinámico, cambiante, con consciencia de sí mismo y con posibilidades de incidir de algún modo en su propio cotidiano.

A continuación se presentan los resultados de un estudio orientado al proceso de configuración de la identidad profesional de personas que dirigen un tipo especial de organizaciones de trabajo: las Organizaciones No Gubernamentales (en adelante ONG). La investigación se desarrolló partiendo de discusiones de la línea de investigación sobre Aspectos Psicosociales del Trabajo como Actividad Económico Productiva, del Grupo de Investigación en Psicología Organizacional y del Trabajo, y de la Maestría en Psicología (Torres, 2010), del Instituto de Psicología, de la Universidad de Valle en Cali, Colombia. Interesó este tipo de organizaciones por el hecho de que han venido constituyendo uno de los sectores significativos hacia el cual el trabajo ha "migrado" (Antunes, 1995), ofreciendo considerables posibilidades de empleo para los profesionales de diversas áreas del conocimiento. Además de esto, resulta importante para la Psicología, comenzar a dar cuenta de la relación persona-trabajo en organizaciones diferentes a las del sector empresarial tradicionalmente estudiadas. La pregunta de investigación que orientó el estudio aquí presentado fue: ¿Cuál ha sido el proceso de construcción de las identidades profesionales de dirigentes de $\mathrm{ONG}$ que se infiere a partir de sus narrativas referidas a sus trayectorias personales y laborales?

\section{Contextualización}

Para caracterizar el tipo de organizaciones en donde se realizó el estudio, es importante mencionar que las ONG hacen parte de una categoría más amplia: las instituciones sin ánimo de lucro $^{3}$, que conforman el conjunto de entidades jurídicas que no hacen parte ni de los organismos del Estado, ni del sector empresarial productivo o de servicios. Generalmente se dedican, según su objeto social, a actividades de beneficio común.

3 El conjunto de entidades sin ánimo de lucro recibe también otras denominaciones, según el ordenamiento jurídico en diferentes países: Tercer sector, sector no lucrativo, sector voluntario, sector no gubernamental, organizaciones de la sociedad civil, organizaciones no gubernamentales, organizaciones de la economía solidaria (Villar, 1998). 
Si bien, esta primera distinción permite diferenciar a las organizaciones del tercer sector, de los otros dos sectores (Público-Estatal y Privado-Lucrativo), cabe anotar que desde el punto de vista jurídico, la figura que permite la normatividad vigente en Colombia es la de "entidades sin ánimo de lucro", dentro de la cual es posible constituir asociaciones, fundaciones, corporaciones o cooperativas en sus diferentes modalidades. Cuando se considera la expresión "Organización No Gubernamental", se hace referencia a una entidad (jurídica) sin ánimo de lucro, que siendo asociación, fundación o corporación, autónomamente decide presentarse ante la sociedad como independiente de las políticas del Estado. Dicho de otro modo, una Organización No Gubernamental (ONG), es una entidad sin ánimo de lucro que lleva a cabo actividades en el campo de los asuntos sociales que en principio son responsabilidad del Estado. Actualmente se cuenta con pocas fuentes documentales ${ }^{4}$ sobre la situación de las ONG en Colombia, el mismo Congreso de la República reconoce: "Las ONG son un fenómeno poco comprendido en Colombia ya que cada quien posee una opinión diferente sobre sus atribuciones, denominación y clasificación dentro de una democracia" (Congreso de la República de Colombia, 2003, p. 1).

Sin embargo, es posible encontrar algunas categorizaciones sobre el campo. Gómez (2003), retomando los aportes Clark (1991), señala siete tipos de ONG considerando su objeto social específico: (1) organizaciones de ayuda humanitaria, (2) agencias populares de desarrollo, (3) organizaciones de desarrollo de base, (4) contratistas de servicios públicos (5) grupos y redes de apoyo, defensa, denuncia e incidencia política, (6) organizaciones de innovación y difusión tecnológica, (7) centros de investigación, estudio y formación. Por otra parte Korten (1990), propone un análisis generacional de ONG a partir de la segunda mitad del siglo XX. La primera generación, centrada en la beneficencia (surgida en los años 50), la segunda (años 60) dedicada a programas de desarrollo, la tercera generación -también llamada generación crítica(años 70), inspirada por las ideas de Paulo Freire y el cambio social, y la cuarta generación, consagrada a la promoción y defensa de los Derechos Humanos a través de la presión política. El estudio aquí presentado, corresponde a aquellas ONG del tipo No. 2, en la clasificación de Clark (1991), y de tercera generación en la propuesta de Korten (1990).

Como en el caso de otras organizaciones de trabajo de tipo empresarial o gubernamental, el mundo de las ONG también ha venido cambiando. A partir de la década de los años 90, varias agencias de financiación europeas han ido retirando el apoyo financiero para centrar sus esfuerzos en otros continentes como el africano y el asiático. La situación tiende a complejizarse pasando de un momento de estabilidad financiera a un contexto en el que también las ONG deben competir por recursos.

En Colombia, mientras que algunas ONG locales han dejado de existir por falta de recursos financieros, otras han iniciado gestiones con organizaciones privadas y del Estado para poder continuar con su objeto social. En este contexto, expertos entrevistados ${ }^{5}$ sostienen que se hace necesario un nuevo tipo de dirigentes que las lideren, más centrados en asuntos de sostenibilidad financiera en lugar de perfiles que enfaticen cuestiones políticas solamente. En ese sentido, sus condiciones técnico-económicas ahora son muy similares a las de las organizaciones tipo empresarial; necesidad de mostrar resultados, competitividad para acceder a convenios, contrataciones temporales, gestión por proyectos, alianzas interinstitucionales, controles administrativos, entre otros.

\footnotetext{
4 Aunque se tenga que a nivel nacional la Confederación Colombiana de ONG (CCONG), dispone de información sobre este sector, es preciso aclarar que las organizaciones aquí referenciadas están agrupadas bajo la categoría jurídica "entidades sin ánimo de lucro” pero sin distinguir cuáles se declaran expresamente ONG o desarrollan programas relacionados con educación popular. En su último informe del año 2011, esta agremiación da cuenta de algunas cifras de sus 53 organizaciones afiliadas, pudiéndose observar aquí la diversidad de entidades, en la que se incluyen varias fundaciones establecidas por grandes empresas colombianas. El lector interesado puede consultar: www.ccong.org.co

5 Grupos de discusión llevados a cabo con integrantes de una institución intermediaria que asesora a más de 70 ONG en Colombia y que reciben el apoyo financiero de una Agencia de Cooperación Europea.
} 


\section{Identidad(es), Self (selves) y el mundo del trabajo}

Partiendo de entender la identidad como la respuesta a la pregunta iquién soy yo?, es propicio acudir a los aportes de George Mead (1973). En su sistema teórico, la mente no es algo substancial, su constitución y contenidos siempre están vinculados al mundo social. En ese sentido la mente de un individuo se origina en un proceso social, en el que se da una conversación interna consigo mismo y desde el cual organiza sus actos como respuestas eficaces ante los estímulos del medio, evaluando y contemplando la respuesta más apropiada en función de su adaptación. Así, desde esta perspectiva se entiende al ser humano como reflexivo, capaz de tomar decisiones considerando lo que observa en su contexto para actuar en él a partir de lo que delibera.

Un proceso de pensamiento fundamental en la concepción de ser humano que propone Mead es la noción de self, definido como la consciencia de sí mismo (Mead, 1973). El self es la capacidad de una persona para ponerse simbólicamente fuera de sí y contemplarse desde otro lugar como si fuera un objeto distinto. Para lograrlo es necesario asumir el punto de vista de otro, es decir, para verse a sí mismo como un objeto, se requiere verse tal como alguien más podría verlo. Esta capacidad de llegar a contemplarnos desde el lugar de otros, se adquiere durante el desarrollo individual y se comienza a configurar desde la niñez, implica dos fases por las cuales el self transita hacia su plena realización. Durante un primer momento, el niño adopta el lugar de otro individuo, con el fin de llevar a cabo ciertas conductas y poder mantener así una relación diádica consistente. En un segundo momento, emerge el "otro generalizado" cuando el niño deberá adoptar no sólo un punto de vista de otro, sino también el de varios que están interrelacionados entre sí, en el marco de una dinámica propia de funcionamiento. De igual manera, para poder sostener una relación consistente con ese colectivo, el niño deberá incorporar el punto de vista de ese grupo organizado para reflexionar sobre sí mismo y valorar la mejor conducta que deberá emprender.

Dado que durante el trayecto de su vida la persona se relaciona con diversos grupos en diferentes espacios y tiempos, se tiene que cada individuo configura varios selves ${ }^{6}$ caracterizados por la reflexión particular a la que llega para responder al medio social concreto en el que interacciona. De este modo se explica la singularidad, considerando las diferentes circunstancias que la persona enfrenta en la vida y ante las cuales discurre, toma decisiones y emprende sus acciones.

Partiendo de los enunciados generales del interaccionismo simbólico de Mead, Scheibe (1995) propone la noción de ser en lugares, como fondo para realizar una psicología del self y de la identidad. En este sentido, para especificar una identidad es necesario recurrir a características con las que es posible ubicar a alguien, dentro de un lugar y un momento concretos, en el marco de una situación social definida. Por ejemplo: edad, género, ocupación etc., conforman un conjunto de marcas de identidad que le permiten a la persona, pero también a sus interlocutores, entablar relaciones sociales consistentes. Algunas de estas marcas son fijas y preceden la existencia del individuo, del mismo modo, otras van cambiando con el trascurrir de la vida personal. Cada quien elige, evaluando la situación, cuántas y cuáles de esas marcas elegir para relacionarse con las demás personas. En esa apreciación que se hace dentro de sí, se puede rastrear el constructo de self, o la consciencia de sí mismo, que se apoya en marcas sociales de identidad para dar cuenta de quién soy, y cómo actuaré en el escenario cambiante del mundo que me rodea. En términos de Scheibe (1995), yo soy un self que tiene una identidad relacionada con un lugar, un espacio y una situación. Este self, en acuerdo con la propuesta de Mead, es una abstracción no conocible directamente por el sujeto. En cambio, sólo se accede a él posteriormente dado que en el proceso reflexivo, las personas simplemente

6 En inglés el término selves corresponde al plural de self. Se opta por mantener los dos términos en el idioma original dado que conceptualmente no corresponden exactamente a traducciones como "El yo" del psicoanálisis o inclusive "El sí mismo" del humanismo. 
lo hacen, sin ser conscientes en ese instante de que lo están haciendo. Dicho de otro modo, la identidad es la respuesta a la pregunta "iQuién soy yo?", pero siempre considerando las coordenadas espacio y tiempo; podría decirse quien soy dependiendo del lugar que ocupo en situaciones particulares; mientras que el self es la reflexión que hago sobre mí mismo, en relación con el lugar social que ocupo bajo esas mismas coordenadas. Aunque cada una de estos dos aspectos -self e identidad-aluden a dimensiones diferentes (pero articuladas) del psiquismo humano, se presenta una relación dialéctica entre estos dos. Para Scheibe la identidad es, de alguna manera, una presentación social del self en un lugar, o mejor, de una parte de él en un momento y lugar específicos. Así mismo, a un self pueden corresponder, en el tiempo, diferentes identidades, según el momento, la época, el contexto y la situación específica.

De otro lado, Ciampa (1987), retomando los aportes del interaccionismo simbólico, de la teoría de papeles y de la dialéctica materialista, propone una concepción de identidad como metamorfosis en el transcurso de la historia particular de las personas. En esta perspectiva se destaca el carácter dinámico de la identidad, diferenciándola de su concepción estática, como la no-metamorfosis o cristalización de la misma. De ese modo se acude a caracterizar la identidad como un proceso dialéctico de cambio constante en las personas, permeado por múltiples aspectos sociales, económicos y culturales propios de un momento histórico particular, incluyendo los propios "auto" determinantes del individuo. Habría que reconocer entonces desde esta propuesta, las condiciones de producción de personajes, refiriéndose a las historias de vida como contextos en los que las personas se re-crean a sí mismas y son a partir de lo que hacen.

Ante todo Ciampa (1987) reitera su interés por superar la concepción estática de la identidad, basada en las atribuciones que los demás nos hacen como una substancia que permanece en el tiempo. Ofrece las categorías actividad, conciencia e identidad como fundamentales para el estudio del hombre por parte de la Psicología Social (Bazilli et al., 1998). Así, se insiste en que no somos algo en particular (por ejemplo una substancia o esencia aprehensible), sino que nuestro ser puede pensarse a partir de lo que hacemos, incluso en diferentes escenarios en los que participamos a lo largo de nuestra trayectoria de vida. La teoría de Ciampa permite caracterizar la identidad como proceso y resultante histórico a la vez, en el que convergen determinantes socio-históricos de la identidad, la permanente autodeterminación individual y una dinámica de constante cambio.

En un análisis sobre los impactos de la globalización en el mundo de los negocios y del trabajo, Malvezzi (2001) comenta cómo las nuevas dinámicas han configurado otro escenario, en el que las identidades profesionales tienden a dejar de ser estáticas o definitivas, referenciadas por tareas predecibles. En lugar de ello, el trabajador actualmente, en situaciones complejas y temporales, mediadas por resultados esperados, está continuamente retado por la velocidad y diversidad de ambientes para crear competencias organizacionales y sociales para la creación de tareas fluidas. Debe administrar sus propios recursos ocupacionales personales a través de procesos de reflexividad. "En ese sentido, la capacidad de administrar su identidad se torna una competencia fundamental para su sobrevivencia profesional” (Malvezzi, 2000, p. 1). Para dar cuenta de la identidad profesional habría que responder a la pregunta "iquién soy yo como persona que trabaja?", acudiendo al conjunto de predicados que constituyen la singularidad del individuo, dejando entrever las semejanzas y diferencias en relación con los otros, es decir, una singularidad construida en relación con los demás a lo largo de la trayectoria de las personas y en un proceso de constante autorreflexión orientado a la movilidad profesional. Mientras en la época preindustrial el artesano era un trabajador que necesitaba de habilidades motoras y de creatividad, la industrialización lo transformó en operario que requería solamente habilidades motoras. En la empresa flexible, propia de la era global, el trabajador se caracteriza por varias facultades: busca el consenso entre argumentos, valora la crítica como la clave para la búsqueda de la certeza, compara entre diferentes criterios 
para valorar los diferentes sentidos de una situación, considera diferentes escenarios en los que los eventos se relacionan de diferentes maneras dado que el ambiente es incierto y ambiguo, conoce las relaciones entre variables para revelar cambios en las rutinas, estima el aporte de sus competencias al logro de resultados.

\section{El posicionamiento discursivo como indicador de selves}

Luego de mencionar las bases conceptuales en las que se apoyó el estudio, resulta ahora primordial referirse a la noción de posicionamiento propuesta por Davies y Harré (1990): "El posicionamiento, como lo usaremos es el proceso discursivo donde los selves se localizan en conversaciones en las que participantes, observable y subjetivamente coherentes, conjuntamente producen argumentos" (p. 5). Estos autores reconocen la ambigüedad de la pregunta "iquién soy yo?" y la consideran un producto de prácticas discursivas. De este modo, al usar las conversaciones como una fuente para entender a las personas, centran sus esfuerzos en analizar la multiplicidad de selves coexistentes y posiblemente contradictorios, pero susceptibles de ser aprehendidos, en un mismo sujeto en el contexto particular de un diálogo con otro. Al caracterizar la posición de un sujeto se estará dando cuenta de cuál es la visión del mundo que tiene desde el lugar o lugares en que se sitúa.

Teniendo en cuenta que las historias pueden tener diversas versiones, cada persona tendria varios selves, y para actuar racionalmente las contradicciones deben ser trascendidas, resueltas o ignoradas ya que "luchamos con diversas experiencias para producir una historia de nosotros mismos que sea unitaria y consistente" (Davies \& Harré, 1990, p. 15). En cuanto a las dinámicas del posicionamiento, los autores distinguen entre, posicionamiento interactivo (ubicar a otra persona) y reflexivo (ubicarse a sí mismo). Al contar fragmentos autobiográficos (por ejemplo anécdotas), el hablante se posiciona a sí mismo y a otras personas que no necesariamente están de acuerdo con esa ubicación asignada. Como ejemplo de análisis del posicionamiento y su relación con el self, Davies y Harré muestran que en las narrativas feministas (de corte posestructuralista), se asumen los diferentes selves en las personas (sobre todo las referidas a mujeres: tradicionales/modernas), evocando la capacidad de elegir quién se desea ser. Se insiste en la posibilidad que tiene cada persona de (auto) determinar su self más allá del rol tradicional que le correspondería asumir, abriendo así la posibilidad de producirse discursivamente a uno mismo o a otro en el marco de un proceso de interacción.

La utilización de la noción de posicionamiento, en la perspectiva de Davies y Harré, resulta crucial para dar cuenta de la identidad - o la respuesta a la pregunta iquién soy yo? inferida a partir de los relatos de las personas sobre sus trayectorias personales y laborales. En ese sentido, con el estudio presentado, se caracteriza el proceso de configuración de la identidad de un grupo de personas, partiendo de sus relatos autobiográficos, dejando entrever el lugar en el que se ubican a sí mismos y a los demás.

\section{Método}

Utilizando entrevistas en profundad, se trabajó con personas de amplio recorrido en el tercer sector y en particular con ONG, ocupando actualmente cargos propios de nivel gerencial. Para caracterizar a los dirigentes de ONG (D-ONG), se aplicó el criterio de sujetos tipo (Hernández, Fernández \& Baptista, 2002) ubicados en cuatro ciudades colombianas en las que existe presencia de entidades internacionales que ofrecen cooperación al desarrollo. 
Para el análisis de la información, se optó por la técnica de análisis de contenido desde la perspectiva de Bardin (2002), definiendo como unidades de registro el objeto o referente (eje temático sobre el cual se organiza el discurso), el personaje (aquellos actores con los que los entrevistados han interactuado en su cotidiano) y el acontecimiento (los sucesos o eventos que perfilan su trayectoria). En cuanto a las reglas de enumeración se definieron la dirección (para dilucidar algunos de los posicionamientos) y la co-ocurrencia (identificación de varias unidades de registro al mismo tiempo).

De manera complementaria para facilitar el procesamiento de la información, se utilizó el recurso de la técnica de líneas narrativas (Spink, 2000), definida como una valiosa posibilidad de esquematizar los contenidos de las historias, en orden "cronológico", mencionados en el transcurso de las entrevistas, dado que en éstas no se narran los hechos de manera organizada conforme ha sucedido en el tiempo.

El modo en que se operacionalizó la información recogida y dispuesta en un texto, se realizó, en primer lugar, detectando mediante análisis de contenido, los referentes temáticos, los personajes y los acontecimientos relatados conforme se desarrolló cada entrevista. En segundo lugar, los acontecimientos fueron ordenados cronológicamente en líneas narrativas. De esta manera, los resultados que se presentan, dan cuenta de lo relatado por los entrevistados en el marco de cinco grandes fases, que conforman en sí mismas, el proceso de configuración de su identidad.

\section{Los relatos organizados en líneas narrativas}

A continuación se presentan los resultados encontrados luego del análisis de las entrevistas realizadas con los D-ONG. Dado que los acontecimientos relatados se ordenaron cronológicamente, en el siguiente apartado se muestran, agrupados, dentro de cinco grandes fases que fueron detectadas como patrón general en cada una de las entrevistas aplicadas. Cada fase es brevemente explicada y ejemplificada con un trecho significativo de alguno de los entrevistados.

Etapa I: Influencias previas. Entendida como los aspectos que inspiraron a los D-ONG en sus años de infancia y adolescencia, para posicionarse, en principio ideológicamente. En términos cronológicos correspondería a una época temprana, en la que ellos aún no se vinculan con alguna modalidad de trabajo en el sentido económico. Sin embargo es aquí en donde se dan los primeros procesos de identificación a través de sus procesos de socialización. Por ejemplo:

Yo salí así porque mi papá me ponía a escuchar discursos de Gaitán, del negro Gaitán ${ }^{7}$, cuando tenía como siete u ocho años y yo no entendía... Después me di cuenta que el partido liberal no era ni revolución ni era cambio, de eso me di cuenta muy rapidito... Cuando tenía trece años, en mí particularmente influyeron mucho dos padres de la teología de la liberación, ese es el momento clarito (Sujeto n. 2).

Etapa II: Trabajo voluntario. Especificada como un momento en el que los sujetos inician algún tipo de actividad productiva, reconocida socialmente en un contexto particular, sin que ello implique alguna remuneración económica, generalmente en contextos denominados "sin ánimo de lucro". Aquí se ubican las acciones motivadas por los primeros referentes ideológicos. Se mencionan las labores realizadas de cuenta propia en los diferentes contextos, acompañados de personas muy cercanas afectivamente:

7 Se refiere al popular líder del partido liberal durante la década de 1940 en Colombia. Su asesinato desencadenó una revuelta conocida en la historia del país como el bogotazo. 
Yo empiezo a digamos a promover otro tipo de cosas ya más desde mi voluntarismo llamémoslo así, y ahí es que yo con todos esos referentes de "El Segundo Sexo" y unas mujeres que yo empiezo a ver en esos barrios... armamos unas semanas culturales... procesos de contribuir al crecimiento de las mujeres, pero muy individualmente. Se puede decir que... lo estaba haciendo yo, no estaba respaldada por ninguna institución... crear ese grupo de mujeres no hacía parte de mi trabajo, o sea eso fue a partir de mi relacionamiento con ellas y de los vínculos que voy estableciendo con la gente del barrio... era pues una cosa que fue muy voluntaria de parte de mía y por otro lado muy espontánea (Sujeto n. 1).

Etapa III: Institucionalización. Caracterizada como el proceso a través del cual la persona menciona un primer ingreso en relaciones de trabajo en el sentido económico-productivo, mediadas por normatividad laboral vigente. En esta etapa los sujetos, se vinculan jurídicamente a alguna forma organizativa o su llamado "grupo de amigos" se transforma en una entidad oficialmente instituida. Aquí se menciona una manera más "racional" de realizar el trabajo de campo. Se encuentran los factores externos que facilitaron una vinculación al mundo formal del trabajo: "Somos un grupo de amigas que trabajamos con mujeres, y va pues produciéndose durante los 80s, un proceso de institucionalización porque empieza a darse la solicitud de trabajos para organizaciones" (Sujeto n. 3).

Etapa IV: Ciclos críticos. Corresponde al conjunto de eventos caracterizados por el juicio evaluador de la persona en su contexto de trabajo. Aquí se ubican las rupturas con diferentes actores: amigos de militancia, opciones laborales, organizaciones, formas de pensar, prácticas, entre otros. Sería la fase en que la persona se sostiene en algo, emergen los arraigos, los apegos, la insistencia para conservar algo: un lugar, una idea, una posición. Es el momento de la valoración de su lugar como dirigente, de la confrontación con los planteamientos de los otros y su consiguiente triunfo. La persona toma decisiones, manifiesta tomar partido en situaciones que no necesariamente comparte. Se mencionan conflictos, expresados en rupturas:

Yo me fui del país, casi 5 años, entonces me fui, de una organización que era eminentemente marcada por el voluntarismo, y volví a una institución en plena crisis... transitar del trabajo voluntario a las reglas del juego del mundo laboral. Entonces eso fue pues, muy duro, fue un momento muy duro, muchas personas salieron... lo que me toca es asumir ese nuevo tránsito, a una institución realmente, pasar de un colectivo a ser una institución, entonces es en ese momento crítico que regreso y ahí pues, con un trabajo muy colectivo vamos en red haciendo el proyecto (Sujeto n. 3).

Etapa V: Consolidación. Entendida como el momento actual en el que los sujetos se desempeñan. No necesariamente es una etapa posterior a la anterior, se plantea la distinción para efectos puramente ilustrativos, resaltando el hecho de que, en el momento de realizar la entrevista, la persona delimita un lugar ocupado, producto de una relación dialéctica entre diferentes elementos psicosociales, configurados de una manera tal, que producen un ser sólo aprehensible temporalmente. Aquí emergen relatos con los que el entrevistado justifica ciertas posiciones, argumenta a su favor las decisiones tomadas para mantener su lugar en relación con varios otros:

Yo soy el tercer o cuarto gerente y ha habido mucha oposición de algunos asociados, pues no sé si por el estilo de trabajo o por varias cosas. Pero afortunadamente, modestia aparte, en los dos años que llevo, es en donde se ha estructurado más la cosa, donde no hay cuentas por pagar, donde tenemos el mayor número de proyectos y de todas maneras, pues, sí quise ser gerente (Sujeto n. 7).

En el cuadro n. 1, se propone un esquema que ilustra el proceso de configuración de la identidad de los D-ONG entrevistados, a partir de las líneas narrativas, inferidas de sus relatos sobre su vida personal y laboral. En la columna "Marcas de Identidad" se relacionan seis categorías que funcionan como coordenadas identitarias (Scheibe, 1995): momentos de vida o etapas del ciclo vital, lugares físicos ocupados, las actividades u ocupaciones, las relaciones en términos de roles, los personajes - naturales o institucionales - con quienes se han entablado 
relaciones y, finalmente, los referentes discursivos en los que se posicionan. En la fila denominada "Etapas" se enumeran las fases por las que han transcurrido los entrevistados en orden cronológico. Es importante aclarar que cada una de esta etapas no tiene fronteras claramente distinguibles, pues sólo representan un esquema para caracterizar un proceso de metamorfosis de la identidad (Ciampa, 1987). De este modo, se ilustran las trayectorias de los D-ONG, considerando aspectos tanto laborales como de otras dimensiones de su vida.

Cuadro 1: Caracterización de las líneas narrativas por etapas y marcas de identidad

$\begin{array}{llllll}\text { Etapas: } & \text { I } & \text { II } & \text { III } & \text { IV } & \text { V } \\ \text { Marcas de } & \text { Influencias } & \text { Trabajo } & \text { Institucionalización } & \text { Ciclos críticos } & \text { Consolidación-actual } \\ \text { identidad: } & \text { previas } & \text { voluntario } & & & \end{array}$

\begin{tabular}{|c|c|c|c|c|c|}
\hline \multicolumn{6}{|c|}{ Momentos de vida } \\
\hline & $\begin{array}{l}\text { Niñez o } \\
\text { adolescencia }\end{array}$ & Juventud & Adultez joven & Adultez & Adultez madura \\
\hline \multicolumn{6}{|c|}{ Espacios o lugares } \\
\hline & $\begin{array}{l}\text { Hogar, barrio, } \\
\text { comunidad, } \\
\text { escuela, iglesia }\end{array}$ & $\begin{array}{l}\text { Barrio propio, } \\
\text { barrio de los } \\
\text { que reciben la } \\
\text { ayuda }\end{array}$ & $\begin{array}{l}\text { Instalaciones de la } \\
\text { ONG }\end{array}$ & $\begin{array}{l}\text { Instalaciones de } \\
\text { la ONG }\end{array}$ & $\begin{array}{l}\text { Instalaciones de la ONG y } \\
\text { otras instituciones }\end{array}$ \\
\hline \multicolumn{6}{|l|}{ Actividades } \\
\hline & $\begin{array}{l}\text { Aprendiz de } \\
\text { ideologías }\end{array}$ & $\begin{array}{l}\text { Trabajo } \\
\text { voluntario, } \\
\text { orientado hacia } \\
\text { la ayuda a otros }\end{array}$ & $\begin{array}{l}\text { Trabajo asalariado, } \\
\text { orientado hacia la } \\
\text { ayuda a otros }\end{array}$ & $\begin{array}{l}\text { Trabajo } \\
\text { asalariado, } \\
\text { orientado hacia } \\
\text { la dirección de } \\
\text { la ONG }\end{array}$ & $\begin{array}{l}\text { Trabajo asalariado, } \\
\text { orientado hacia la dirección } \\
\text { y sostenimiento de la ONG }\end{array}$ \\
\hline \multicolumn{6}{|l|}{ Relaciones } \\
\hline & $\begin{array}{l}\text { Hijo, hermano, } \\
\text { alumno }\end{array}$ & $\begin{array}{l}\text { Hijo, hermano, } \\
\text { alumno, } \\
\text { compañero }\end{array}$ & Compañero & $\begin{array}{l}\text { Dirigente, } \\
\text { madre o padre }\end{array}$ & Dirigente, madre o padre \\
\hline \multicolumn{6}{|l|}{ Personajes } \\
\hline & $\begin{array}{l}\text { Familiares, } \\
\text { profesores, } \\
\text { sacerdotes, } \\
\text { amigos, } \\
\text { beneficiarios de } \\
\text { la ayuda }\end{array}$ & $\begin{array}{l}\text { Amigos, } \\
\text { familiares, } \\
\text { beneficiarios de } \\
\text { la ayuda }\end{array}$ & $\begin{array}{l}\text { Compañeros, } \\
\text { entidades donantes, } \\
\text { (ONG europeas, } \\
\text { Organizaciones } \\
\text { Gubernamentales } \\
\text { nacionales), ONG } \\
\text { empleadoras }\end{array}$ & $\begin{array}{l}\text { Compañeros } \\
\text { disidentes, otras } \\
\text { ONG surgidas a } \\
\text { partir de las } \\
\text { crisis }\end{array}$ & $\begin{array}{l}\text { Equipo de trabajo, junta } \\
\text { directiva, agencias y } \\
\text { personas donantes, } \\
\text { instituciones, redes inter- } \\
\text { institucionales }\end{array}$ \\
\hline \multicolumn{6}{|l|}{ Referentes } \\
\hline & $\begin{array}{l}\text { Ideologías de } \\
\text { cambio social }\end{array}$ & $\begin{array}{l}\text { Capacitación a } \\
\text { de población }\end{array}$ & $\begin{array}{l}\text { Temas concretos de } \\
\text { proyectos }\end{array}$ & $\begin{array}{l}\text { Eficiencia en el } \\
\text { trabajo }\end{array}$ & $\begin{array}{l}\text { Gestión de recursos y auto- } \\
\text { evaluación de proyectos }\end{array}$ \\
\hline
\end{tabular}

beneficiaria

Al hacer un análisis de la información en sentido horizontal, se revelan acontecimientos, personajes y referentes mencionados en común por parte de los sujetos, dentro de cada una de las cinco grandes etapas, que inician desde la infancia o adolescencia, hasta el momento presente en el que se realizan las entrevistas. Esto fue posible a través de la utilización en el análisis de contenido de la regla de enumeración "co-ocurrencia", que permite localizar la presencia simultánea de las unidades de registro a lo largo de todas las entrevistas realizadas, para así llegar a los aspectos comunes que caracterizan las trayectorias personales y profesionales de los D-ONG. 
Como se logra apreciar, se presenta una tendencia hacia la complejidad de las actividades que realizan los sujetos, pasando de un momento en el que acogen una idea de ayuda dirigida a otros, hacia una materialización de ese pensamiento en una acción concreta, que en un principio no es remunerada ni regulada externamente, pero que más adelante se desarrolla en el marco de un sistema que implica otros aspectos además de la ayuda a otros, tales como el modo de hacerlo, los recursos necesarios, los tiempos y la justificación ante pares y externos. Respecto a los lugares, se observa que el tránsito ha sido desde espacios en los que los sujetos están supeditados a influencias, por las condiciones de sus procesos de socialización, hacia lugares en los que ellos mismos preparan las condiciones para incidir en la vida de otras personas.

Utilizando la noción de ser en lugares de Scheibe (1995), a través de marcas de identidad, se tiene que en el caso de los D-ONG entrevistados, es posible ubicar sus identidades en función de las coordenadas tiempo y espacio, en donde son reconocidos socialmente por el lugar que ocupan en relación con los demás. Así, los sujetos son una identidad particular dependiendo del lugar ocupado en un momento específico, es decir; son reconocibles a partir de marcas de identidad, por ejemplo ser hijo que aprende en el hogar durante la infancia, o ser dirigente que planifica en la ONG durante la adultez. De este modo, deberá entenderse que estas marcas no son las mismas ni en el transcurso del tiempo, ni en los diferentes espacios que la persona ocupa. En este sentido, se observa que los sujetos viven en constante cambio (Ciampa, 1987), actualizando permanentemente su identidad específica, en este caso ligada al trabajo. Este ser en constante cambio, es aprehensible, se revela, se observa a partir de sus actividades, como un ser definido por lo que hace, pasando de ser una persona que sólo aprende, a ir siendo progresivamente alguien que le dirige el trabajo a otros

Entre los personajes (personas o instituciones) mencionados por los sujetos a lo largo de las etapas distinguidas, se tiene que durante los dos momentos iniciales, los sujetos se refieren a aquellas personas que incidieron en su socialización durante la infancia o adolescencia, quienes les alentaron a que ingresaran al medio de los movimientos sociales. Durante la segunda etapa, los sujetos aluden a los beneficiarios de la ayuda ofrecida en el trabajo voluntario. A partir de la tercera etapa, los sujetos mencionan aquellas personas naturales o jurídicas que les ofrecieron la posibilidad de que su trabajo sea remunerado. En la siguiente etapa, se puede observar cómo los sujetos mencionan particularmente con nombre propio, a las personas con las que se confrontan discutiendo puntos de vista diferentes en torno a las condiciones en las que realiza el trabajo. Es aquí en donde se presentan las "crisis" institucionales (en el caso de ONG conformadas por algunos entrevistados) o de diferencias que algunos de los sujetos mencionan en relación con la $\mathrm{ONG}$ para cual trabajaban en ese momento. En la etapa presente, los sujetos mencionan a nivel interno a los "equipos de trabajo", mientras que a nivel externo aluden a personas naturales y jurídicas con las cuales gestionan recursos para sostener la ONG que dirigen.

En este aspecto, se observa un movimiento de relaciones personales definidas por el afecto, con una tendencia hacia relaciones personales mediadas por la razón instrumental ligada a la conservación del trabajo, sin dejar de mencionar que en ese tránsito, ocurren rupturas en relaciones de amistad, generadas por las diferencias manifiestas. En este sentido, se tiene que a partir de la institucionalización del trabajo, los entrevistados amplían sus redes sociales incorporando en adelante, relaciones con personas naturales y jurídicas en dinámicas no sólo de ayuda a otros sino también de orden funcional.

El análisis de los personajes mencionados por los entrevistados durante el transcurso de las diferentes etapas, permite rastrear la emergencia del self a partir de la noción del otro generalizado. Retomando a Mead, es pertinente mencionar que el ser humano es entendido como reflexivo y capaz de tomar decisiones deliberadamente razonando sobre lo que percibe en su medio para actuar en este. Sin embargo, se requiere también aclarar que para Mead, aunque el self hace que el individuo sea más eficiente para el conjunto de la sociedad respondiendo a 
las expectativas del grupo, éste no siempre tiene que aceptar el colectivo tal y como es. Por el contrario, las personas pueden cambiar las condiciones del grupo en el que participan gracias a su capacidad de pensar, incluso oponiéndose al mismo, pero proponiéndole un escenario más favorable para todos los implicados. Al respecto Mead (1973) comenta:

La única forma en que podemos reaccionar contra la desaprobación de la comunidad entera es estableciendo una clase superior de comunidad que, en cierto sentido, supere en número de votos a la que conocemos. Una persona puede llegar al punto de ir en contra de todo el mundo que le rodea; puede ella sola levantarse contra el mundo. Pero, para hacer tal cosa, ha de hablarse a sí misma con la voz de la razón. Tiene que abarcar las voces del pasado y del futuro. Esta es la única forma en que el self puede lograr una voz que sea mayor que la voz de la comunidad (p. 196).

De este modo, se observa la posición que adoptan los entrevistados en relación con el colectivo en el que participan, ya que ante la disyuntiva entre continuar como grupo informal o institucionalizarse, logran visualizar y proponer, dadas las circunstancias, un escenario futuro que sería mejor para todos. Ahora bien, es gracias a esa visión de futuro que logran establecer, que los demás integrantes empiezan a reconocerlos como líderes, aunque algunos otros integrantes no estén de acuerdo con el camino trazado y finalmente decidan retirarse.

En lo concerniente a los referentes, los sujetos en su primera etapa mencionan los discursos que acogieron durante sus procesos de socialización, durante la niñez o adolescencia, y que están relacionados con ideologías de cambio social: feminismo, teología de la liberación, la revolución. Durante la segunda etapa, en sus espacios de trabajo voluntario, se aprecia en general un discurso centrado en un conocimiento que se ofrece a los beneficiarios, nombrado como procesos de "capacitación" o "empoderamiento". Luego, cuando el trabajo se ha instituido, se encuentran alusiones a temas concretos en los que se inscriben los proyectos; incidencia, autoconstrucción, liderazgo, cooperativismo, entre otros. En la siguiente etapa se tiene que, dados los conflictos entre formas de concebir y realizar el trabajo, se mencionan aspectos del cumplimiento y control de las actividades programadas en los proyectos que administra la ONG. Finalmente se encuentra que en la etapa en que se encuentran los entrevistados, el discurso gira alrededor de las funciones directivas que en términos generales, comprenden la atención de dos niveles organizacionales: la dimensión externa, relaciones interinstitucionales y gestión de recursos, y la dimensión interna, referida como el "acompañamiento al equipo de trabajo".

Sobre los referentes en la etapa actual de los dirigentes D-ONG, se observa un cambio que pasa de ser lo referido a la ayuda a los demás, con justificaciones ideológicas sobre igualdad social, equidad de género o participación política, hacia discursos más centrados en la eficiencia en el trabajo y posteriormente en la gestión de recursos para el sostenimiento de la ONG y su misión institucional. Este giro en los referentes ideológicos de los entrevistados podría estar asociado a dos elementos contextuales. De un lado Dent y Whitehead (2002), mencionan la tendencia global hacia la que se dirige la valoración del trabajo profesional, y que sugiere una cultura del rendimiento en la que los profesionales deben incorporar en sus parámetros de desempeño, los resultados organizacionales que el medio les exige. En otras palabras, incluso los D-ONG se ven presionados a cumplir con resultados concretos sobre los cuales se estima su desempeño. De otro lado, Castillo (2003) menciona entre otros cambios en el mundo de la cooperación internacional, la tendencia hacia la disminución de recursos para el apoyo de ONG locales. En ese sentido, se tiene pues, un tránsito histórico que parte de un contexto en el que se valoraba la presencia de ONG acompañando poblaciones beneficiarias, hacia un momento -presente- en el que se espera de las ONG y particularmente de sus dirigentes, el cumplimiento de metas en tiempos concretos y con recursos que requieren más controles administrativos. Es decir, mientras en los años 70 se financiaban organizaciones que alentaban movimientos sociales con reivindicaciones políticas, actualmente las entidades financiadoras tienden a concentrar su apoyo a proyectos puntuales, cuyos resultados sean visibles, lo que 
explica la orientación de los D-ONG hacia privilegiar discursos de eficiencia. Este hallazgo coincide con varios de los cuestionamientos críticos elaborados por analistas del mundo de la cooperación para el desarrollo, cuando se refieren a la priorización de la gestión de recursos financieros en el ámbito de las ONG. Al respecto vale la pena mencionar a Eade (2002) en una reflexión sobre la supervivencia institucional:

El resultado es que las ONG pueden adaptarse exitosamente a un mercado cambiante para asegurarse un continuo suministro de fondos, pero ello a costa de sacrificar su tarea de facilitar de manera efectiva un cambio social radical, o de representar alternativas reales al paradigma dominante (p. 12).

Retomando a Malvezzi (2001), habría que reconocer el dinamismo de las identidades de los dirigentes entrevistados, considerando que para responder a la pregunta iquién es ese trabajador?, de ninguna manera podría esperarse que la respuesta fuera resuelta sin acudir al devenir histórico ilustrado a través de las etapas mencionadas. En cambio, se tendría que afirmar entonces que estos sujetos han ido siendo, a lo largo de sus trayectorias, personas que han reflexionado sobre sus posiciones en relación con los demás y que de manera autónoma (dentro de su contexto), han tomado decisiones sobre los lugares que ocupan. Particularmente en relación con el trabajo, estos sujetos han estado inmersos en situaciones de confrontación de posicionamientos incluso antes de vincularse formalmente en el mundo profesional. En otras palabras, sus vínculos con los contenidos de su trabajo profesional comienzan a conformarse desde los primeros espacios de relacionamiento en la familia, el barrio y la escuela. Entendiendo esto, habría que buscar las raíces de la carrera profesional en los procesos de socialización que comienzan en la infancia y adolescencia para visualizar el movimiento identitario y la emergencia del self.

\section{A modo de conclusión}

Luego de llevar a cabo el estudio aquí presentado, es posible afirmar que para caracterizar la identidad es necesario pensar no en términos estáticos, sino en una dinámica en la que el sujeto reflexiona sobre sí mismo y el lugar que va ocupando ante los demás dadas las condiciones sociales del momento en su medio. Dicho en otras palabras, los D-ONG no son, en cambio, han ido siendo a través de una construcción social e histórica de sí mismos, personas que se han relacionado con el contenido de su trabajo desde sus años de infancia, acudiendo a premisas ideológicas y con miras en su realización personal. En este proceso, los D-ONG han reflexionado sobre sus marcas de identidad, pasando por situaciones de rupturas y acogidas de referentes y personajes en un movimiento que no escapa a las nuevas realidades globales del mundo de los negocios que actualmente les exige, entre otras cosas, acoger los aspectos propios de la cultura del "managerialism": flexibilidad, reflexividad pragmática, trabajo en equipo, aprendizaje en el largo plazo, actualización constante, emprendedor y orientado hacia el mercado. Para llegar a su posición actual y mantenerse en ella, dadas las condiciones del contexto, los D-ONG se desenvuelven en una dinámica de posicionamientos y reposicionamientos en constante interacción con otros generalizados.

Mientras que algunos de los entrevistados han desarrollado su carrera en el tránsito del grupo de amigos hacia la institucionalización, otros la han realizado entre varias organizaciones del mismo sector. En cualquiera de los casos mencionan rupturas y cambios en sus relaciones (Sennett, 2000) a partir de la diferenciación de posicionamientos ante otros, distinguiendo así su singularidad. El hallazgo de indicios de reflexividad a lo largo de las trayectorias de los DONG, permite dar cuenta de que su carrera en este contexto, no empieza con el primer contrato formal de trabajo, sea cual fuera la modalidad de trabajo (Rentería, 2001) o 
vinculación jurídico-administrativa. En este caso, es posible observar una continuidad que se empieza a configurar desde los procesos de socialización en la infancia y la adolescencia, tal como lo narran ellos mismos. En este orden de ideas, es posible entonces consolidar la idea de que en el actual contexto, las carreras profesionales no necesariamente se inscriben en fronteras tan claramente diferenciadas (Malvezzi, 2001).

Ahondando en la caracterización de la trayectoria de los D-ONG, es posible ilustrar sus dinámicas metafóricamente como en una espiral, considerando que en el paso de una etapa a otra, el sujeto incorpora nuevos recursos, en lo que Malvezzi (1999) menciona como insumos de trabajo. En ese orden de ideas se puede apreciar con el avance de su carrera, cómo la persona va ampliando sus repertorios interpretativos y de acción, se hace profesional, gana un reconocimiento social y económico, justifica de manera más amplia sus acciones. Contrario a lo que podría pensarse, dadas las condiciones económicas en el sector no lucrativo, las posibilidades de crecimiento profesional serían limitadas. Sin embargo, los resultados muestran a modo de una trayectoria en espiral, cómo estas personas se sostienen y avanzan en su proceso personal de construcción de su propia identidad.

Entendiendo cómo los D-ONG han ido reflexionado, expresado y confrontado sus posiciones desde temprana edad y a lo largo de sus trayectorias, es claro comprender cómo generan su permanencia en el sector de las ONG, ejecutando, culminando y reiniciando nuevos proyectos de intervención social a través de los cuales, generan su fuente de sostenibilidad, aunque es claro que lideran organizaciones dependientes desde el punto de vista financiero. En otras palabras, los D-ONG han ido capitalizando recursos argumentativos plasmados en sus repertorios de interpretación y de acción indistintamente del lugar de trabajo ocupado, más allá de su formación profesional, asumiendo desde el cotidiano y desde temprana edad posiciones dirigidas al campo de la labor social. Sería interesante discutir hasta qué punto sus reivindicaciones sociopolíticas son expresión de posicionarse como agentes de cambio, o si de otro modo, han desempeñado de eficientemente ciertos papeles prediseñados en el contexto de los debates sobre el desarrollo social y económico, asunto discutido por Enriquèz (2000), quien sugiere que a pesar de la relativa libertad con la que trabajan los individuos en nuestra época, de todos modos lo hacen en el marco de "autonomías controladas" determinadas por las estructuras organizacionales. En cualquier caso, los resultados sugieren que a pesar del soporte ideológico de su trabajo, los D-ONG reproducen las contradicciones entre capital y trabajo, aspecto evidenciado en su tendencia hacia la incorporación de discursos de eficiencia en su cotidiano.

En consonancia con Ciampa (1987), y asumiendo la identidad como un proceso constante de cambio, para caracterizar el caso de los D-ONG, aquí fue necesario también acudir a sus múltiples determinantes, sociales, económicos y culturales propios del momento histórico, incluyendo los propios - auto - determinantes del individuo. Lo que mostró el estudio es precisamente, cómo los sujetos se han ido posicionando discursivamente frente a estas condiciones contextuales, a partir de reflexiones que hacen individualmente para ir construyendo (se), recreando (se), en cada momento histórico a partir de lo que hacen. En ese sentido, queda bien valorada la propuesta teórica de Ciampa centrada en los tres elementos; los determinantes socio-históricos, la permanente auto-determinación individual y el constante cambio, como una opción importante que permiten dar cuenta del proceso de metamorfosis de los sujetos en su relación con el trabajo. Finalmente, se ha mostrado cómo en el intento de caracterizar la identidad, es posible acudir a una perspectiva alternativa a la de "sujeto cristalizado". En este caso, se ha dado cuenta del proceso de transformación del dirigente de ONG, que empieza a configurarse desde los años de infancia hasta la actualidad, desarrollando sus posicionamientos frente a las marcas de identidad que van afrontando durante sus trayectorias.

Respecto a los modelos de gestión implementados por los D-ONG, aunque estos no se exploraron directamente, se pueden discutir tres elementos que se derivan del análisis del 
proceso de configuración de su identidad. En primer lugar, se encuentra que a partir del momento de la institucionalización (Etapa n. 3), los sujetos muestran un cambio en el referente, que va desde la mención a sus fundamentos ideológicos hacia la priorización del desempeño en el lugar de trabajo como eje central del discurso. En ese sentido, se tiene que los D-ONG han ido ampliando sus repertorios de comprensión de su realidad y ya no solamente consideran importantes los aspectos estrechamente ligados a la labor social, sino que también ahora incorporan elementos de la gestión administrativa. Un segundo elemento, apunta a que los D-ONG, aunque en un principio (durante los años 70 y 80 ) intentaron conformar formas organizativas diferentes a las del sector lucrativo, actualmente tienden a implementar estructuras jerárquicas propias de los modelos empresariales de gestión. Por último, en un tercer elemento, se puede destacar que algunos de los sujetos mencionan que en sus prácticas administrativas, acuden a ciertas acciones que los diferencian de los típicos de estilos gerenciales de la empresa privada-lucrativa. Al respecto, resultaría interesante desarrollar futuros estudios que permitan caracterizar más a fondo el tema de los estilos y modelos gerenciales, considerando la identidad profesional, para continuar profundizando en la compresión psicosocial en este sector. De todos modos es posible apreciar en los D-ONG una concepción ideal de trabajo bajo la modalidad de empleo, dado que actualmente sus esfuerzos están dedicados a la gestión y administración eficiente de recursos, con miras a mantener las condiciones que la financiación internacional facilitó, por varios años en el desarrollo de su carrera profesional.

Como aspecto relevante complementario, la dimensión metodológica del estudio permitió valorar varias posibilidades que ofrecen diferentes técnicas de análisis de información. Resultó muy importante encontrar la amplitud de elementos de los que se puede disponer al analizar un mismo material de estudio (en este caso un texto disponible), a través de diferentes técnicas inspiradas en propósitos diferentes pero no excluyentes. De un lado, el análisis de contenido hace que se visibilicen todos aquellos elementos sobre los cuales los entrevistados organizan sus relatos, permitiendo que el investigador disponga de los aspectos temáticos necesarios. De otro lado, las líneas narrativas ofrecen a su vez, ordenar cronológicamente e ilustrar de manera clara todos aquellos eventos que los sujetos narran y que se pueden contrastar cuando se pretenden rastrear los posicionamientos en una conversación concreta. Si se tiene en cuenta que al inicio del presente estudio se había contemplado solamente el análisis de contenido (en la perspectiva de Bardin), resulta válido afirmar que durante el proceso de investigación, es posible acudir a herramientas no previstas con el fin de complementar el procedimiento y llegar a los análisis esperados de una manera más consistente. Así mismo, se podría también implementar otra técnica para procesar la información encontrada: los mapas conceptuales. De este modo, ya identificadas las etapas por las cuales han transitado los D. ONG, el texto se podría desagregar en cinco columnas correspondiente a cada una de las etapas y así poder visualizar a qué momento de la trayectoria corresponde cada idea expresada.

Finalmente, a partir de los resultados encontrados en el presente estudio, surgen tres inquietudes para profundizar a futuro: ¿Coinciden las etapas de las trayectorias de los D-ONG con la propuesta de Erik Erikson (1988) sobre las etapas del desarrollo psicosocial? iEs posible explicar el fenómeno del liderazgo a partir de del concepto "otro generalizado" de George Mead tal como se observa en los D-ONG? ¿Qué semejanzas podrían establecerse entre los cambios socio-históricos aquí ilustrados y la situación actual de los movimientos sociales en América Latina? 


\section{Referencias}

Antunes, R. (1995). Qual crise do trabalho? In R. Antunes, Adeus ao trabalho? Ensaio sobre as metamorfoses e a centralidade do mundo do trabalho. São Paulo: Cortez/Editora da Uniccamp.

Bardin, L. (2002). Análisis de contenido (3를 ed.). Madrid: Ediciones Akal.

Bauman, Z. (2005). Modernidad líquida. Buenos Aires: Fondo de Cultura Económica.

Bazilli, C., Rentería, E., Duarte, J., Simões, K., Feitosa, L. \& Rala, L. (1998). Interacionismo simbólico e teoria dos papéis: uma aproximação para a psicologia social. São Paulo: EDUC.

Beck, U. \& Beck-Gernsheim, E. (2002). Individualization. London: Sage.

Castillo, O. (2003). La cooperación internacional para el desarrollo. Contexto global. En Fundación para la Cooperación Synergia. Aportes a la cooperación internacional en Colombia (pp. 13-37). Bogotá: Fundación para la Cooperación Synergia.

Ciampa, A. (1987). A estória de Severino e a história da Severina. São Paulo: Brasiliense.

Congreso de la República de Colombia. (2003). Organizaciones no gubernamentales, regulación, control y vigilancia. Programa de Fortalecimiento Legislativo. Oficina de Asistencia Técnica Legislativa. Bogotá, Colombia, 27 de noviembre de 2003. Recuperado en 2 de febrero, 2010, de http://secretariasenado.gov.co/estudiosARD/023\%ONG.

Clark, J. (1991). Democratizing development: the role of voluntary organizations. West Hartford: Kumarian Press.

Davies, B. \& Harré, R. (1990). Positioning: the discursive production of selves. Journal for the Theory of Social Behavior, 20 (1): 43-63, 1990

Dent, M. \& Whitehead, S. (2002). Managing professional identities: knowledge, performativity and the "new" professional. Londres: Routledge.

Eade, D. (2002). Prologo. In Intermón Oxfam, Desarrollo, ONG y sociedad civil. Barcelona: Intermón Oxfam. Recuperado en 9 de septiembre, 2010, de http://www.ebpdn.org/download/download.php? table $=$ resources\&id $=1043$.

Erikson, E. (1988). El ciclo vital completado. Buenos Aires: Paidós

Enriquéz, E. (2000). O indivíduo preso na armadilha da estrutura estratégica. In F. Motta \& M. de Freitas, Vida psíquica e organização. Rio de Janeiro: Editora da FGV.

Gómez, M. (2003). Las organizaciones no gubernamentales de desarrollo (ONGD). In Fundación para la Cooperación Synergia (Org.). Aportes a la cooperación internacional en Colombia (pp. 13-37). Bogotá: Fundación para la Cooperación Synergia.

Hernández, R., Fernández, C. \& Baptista, P. (2002). Metodología de la investigación. México: McGraw Hill.

Korten, D. (1990). Getting to the $21^{\text {th }}$ century: voluntary action and the global agenda. West Hartfort: Kumarian Press.

Malvezzi, S. (1999). O agente económico reflexivo. Desarrollo y capacitación, 49, 16-19.

Malvezzi, S. (2000). A construção da identidade profissional no modelo emergente da carreira. Organização e Sociedade, 7 (17), 137-143.

Malvezzi, S. (2001). The building of professional identity and the boundaryless careers. [Conferencia no publicada]

Mead, G. (1973). Espíritu, persona y sociedad desde el punto de vista del conductismo social. Barcelona: Paidós Ibérica.

Offe, C. (1995). Trabalho, a categoria sociológica chave? In C. Offe, Capitalismo desorganizado. São Paulo: Brasiliense.

Rentería, E. (2001). El modelo educativo tradicional y los perfiles de competencias según las modalidades y tendencias del trabajo actuales. En AUIP, Vinculación universidad-empresa a través del posgrado (pp. 51-61). Salamanca: AUIP.

Rentería, E. (2008). Nuevas realidades organizacionales y del mundo del trabajo: implicaciones para la construcción de la identidad o del sujeto. Informes Psicológicos, 10, 65-80.

Rentería, E. (2009). De recursos humanos a la psicología organizacional y del trabajo: reflexiones a la luz de las realidades actuales del mundo del trabajo. In M. Aguilar \& E. Rentería (Comp.), Psicología del trabajo y de las organizaciones: reflexiones y experiencias de investigación. Bogotá: Universidad Santo Tomas.

Rentería, E. \& Malvezzi, S. (2008). Empleabilidad, cambios y exigencias psicosociales en el trabajo. Universitas Psychologica, 7 (24), 9-24.

Ribeiro, M. (2009). A trajetória da carreira como construção teórico-prática e a proposta dialética da carreira psicossocial. Cadernos de Psicologia Social do Trabalho, 12 (2), 203-216.

Sato, L. (1997). Astúcia e ambiguidade: as condições simbólicas para o replanejamento negociado do trabalho no chão de fábrica. Tesis de Doctorado en Psicología Social, Instituto de Psicologia, Universidade de São Paulo, São Paulo, Brasil.

Sato, L. (2002). Prevenção de agravos à saúde do trabalhador: replanejando o trabalho através das negociações cotidianas. Cadernos de Saúde Pública, 18 (5), 1147-1166.

Sennett, R. (2000). Street and office: two sources of identity. In W. Hutton \& A. Giddens (Eds.), On the edge: living with global capitalism (pp. 175-190). Londres: Jonathan Cape.

Sennett, R. (2002). A corrosão do caráter: consequências pessoais do trabalho no novo capitalismo. Rio de Janeiro: Record.

Scheibe, K. (1995). Self studies: the psychology of self and identity. Londres: Praeger.

Schein, E. (1982). Dinámica de la carrera empresarial. México: Fondo Educativo Interamericano. 
Spink, M. (2000). Práticas discursivas e produção de sentidos no cotidiano. São Paulo: Cortez.

Torres, C. (2010). Identidad profesional de dirigentes de organizaciones no gubernamentales a partir de sus trayectorias. Tesis de Maestría en Psicología. Instituto de Psicología. Universidad del Valle, Cali, Colombia.

Villar, R. (1998). Defining the nonprofit sector: Colombia (Working Paper of the Johns Hopkins Comparative Nonprofit Sector Project, n. 29) (Lester M. Salamon \& Helmut K. Anheir, Ed.). Baltimore: The Johns Hopkins Institute for Policy Studies. Recuperado en 1 de septiembre, 2011, de http://admcf.com/jhu/pdfs/CNP_Working_Papers/CNP_WP29_Colombia_1998.pdf.

Weick, K. (1982). Psicología social del proceso de organización. Bogotá: Fondo Educativo Latinoamericano.

\section{Endereço para correspondência}

erico.renteria@correounivalle.edu.co,carlos.f.torres@correounivalle.edu.co

Recebido em: 06/03/2012

Revisado em: 09/09/2012

Aprovado em: 11/09/2012 